\title{
Grupo de psicoeducação no transtorno afetivo bipolar: reflexão sobre o modo asilar e o modo psicossocial*
}

\author{
PSYCHOEDUCATIONAL GROUP AND BIPOLAR AFFECTIVE DISORDER: A REFLECTION \\ ON THE ASYLUM AND PSYCHOSOCIAL MODELS
}

\section{GRUPO DE PSICOEDUCACIÓN EN EL TRANSTORNO AFECTIVO BIPOLAR: REFLEXIÓN SOBRE EL MODELO ASILAR Y EL MODELO PSICOSOCIAL}

\section{Sarita Lopes Menezes' ${ }^{1}$ Maria Conceição Bernardo de Mello e Souza²}

\section{RESUMO}

O Transtorno Afetivo Bipolar (TAB), conhecido por sua cronicidade, complexidade e altos índices de morbidade e mortalidade, é uma das principais causas de incapacitação no mundo. Há evidências crescentes de que seu curso pode ser modificado por abordagens psicoterápicas como a psicoeducação, que promove o aumento do funcionamento social e ocupacional, bem como da capacidade de manejarem situações estressantes. Trata-se de um relato de experiência sobre um grupo de psicoeducação para familiares e portadores de TAB, sendo esse pautado pela reflexão dos componentes do paradigma das práticas em saúde mental. A reflexão sobre os modos asilar e psicossocial quanto às concepções de objeto e modo de trabalho, possibilitou situar o grupo no modo psicossocial, pois o conhecimento e a vivência experimentados favorecem a autonomia dos sujeitos, que têm maiores chances de se posicionarem frente às dificuldades que lhes sobrevêm na sua existência-sofrimento.

\section{DESCRITORES}

Transtorno bipolar

Terapêutica

Saúde mental

Enfermagem psiquiátrica

Educação em saúde

\begin{abstract}
Bipolar Affective Disorder (BAD), known by its chronicity, complexity and high rates of morbidity and mortality, is one of the main causes of disability in the world. There is growing evidence that its path can be changed by psychotherapy approaches, such as psychoeducation, which improves one's social and occupational behavior and capacity to deal with stressful situations. This study is an experience report about a psychoeducational group for individuals with $B A D$ and their relatives, which consisted on reflecting upon the components of the mental health practice paradigm. Reflecting upon the psychosocial and asylum ways regarding the conceptions of object and mode of work permitted to locate the group in a psychosocial manner, as knowledge and experience favor the autonomy of the individuals, who have more chances to face the difficulties that occur in their suffering-existence process.
\end{abstract}

\section{DESCRIPTORS \\ Bipolar disorder \\ Therapeutics \\ Mental health \\ Psychiatric nursing \\ Health education}

\section{RESUMEN}

El Transtorno Afectivo Bipolar (TAB), conocido por su cronicidad, complejidad y altos índices de mortalidad y morbilidad, es una de las principales causas de incapacidad en el mundo. Existen evidencias crecientes de que puede modificarse su curso con abordajes psicoterapéuticos, como la psicoeducación, que promueve el aumento del funcionamiento social y ocupacional, así como de la capacidad de manejar situaciones estresantes. Se trata de un relato de experiencia sobre un grupo de psicoeducación para familiares y pacientes de TAB, pautado en la reflexión de los componentes paradigmáticos de las prácticas de salud mental. La reflexión entre los modelos asilares y psicosociales referidas a las concepciones del objeto y modalidad de trabajo posibilitó situar el grupo en el modelo psicosocial, pues el conocimiento y la vivencia experimentados favorecen la autonomía del sujeto, que tiene mayores chances de posicionarse frente a las dificultades que devienen de su existencia y sufrimiento.

\section{DESCRIPTORES \\ Trastorno bipolar \\ Terapéutica \\ Salud mental \\ Enfermería psiquiátrica \\ Educación en salud}

\footnotetext{
* Extraído da dissertação "Grupo de Psicoeducação e suas implicações no cotidiano de portadores de Transtorno Afetivo Bipolar", Escola de Enfermagem de Ribeirão Preto da Universidade de São Paulo, 2009. ${ }^{1}$ Enfermeira. Mestre em Enfermagem Psiquiátrica pela Escola de Enfermagem de Ribeirão Preto da Universidade de São Paulo. São José do Rio Preto, SP, Brasil. sarita_enf@yahoo.com.br ${ }^{2}$ Enfermeira. Professora Doutora do Departamento de Enfermagem Psiquiátrica e Ciências Humanas da Escola de Enfermagem de Ribeirão Preto da Universidade de São Paulo. Ribeirão Preto, SP, Brasil. consouza@cerp.usp.br 


\section{INTRODUÇÃO}

O TAB (Transtorno Afetivo Bipolar) é conhecido por sua cronicidade, complexidade e pelos altos índices de morbidade e mortalidade, caracterizado por episódios de mania ou hipomania, alternados com períodos de depressão e/ou eutimia(1).

Na fase de mania o humor é expansivo ou eufórico, podendo ser irritável e desinibido. Há, também, diminuição da necessidade de sono, inquietação, agitação psicomotora, aumento de energia e de libido. Além disso, é comum ocorrer nessa fase ideias de grandeza, prolixidade, pressão para falar, prejuízo da crítica e aumento da impulsividade. Assim, a conduta social torna-se inadequada, o indivíduo pode ficar indiscreto, invasivo, bem como aumentar o consumo de álcool e/ou outras drogas, aumentar os gastos financeiros e o envolvimento em atividades potencialmente danosas, como dirigir em alta velocidade, promiscuidade sexual e dívidas ${ }^{(2)}$.

Já o quadro de hipomania é descrito como uma mania atenuada, sendo que as alterações devem ser notórias, percebidas por outros. Porém, diferente da mania, não leva ao comprometimento funcional do indivíduo e não pode incluir sintomas psicóticos ${ }^{(2)}$.

O episódio depressivo, por sua vez, é caracterizado, principalmente, por alterações no humor, na psicomotricidade, na cognição e nas funções vegetativas, alterações estas opostas às que ocorrem na mania. Os principais sintomas são: humor depressivo, incapacidade de sentir alegria ou prazer, diminuição da energia, lentificação ou agitação psicomotora, diminuição da libido, apetite e sono, dificuldades de concentração e pensamentos de cunho negativo, podendo ocorrer ideação suicida e/ou sintomas psicóticos $^{(2)}$.

Pode ocorrer, também, o episódio misto, no qual o paciente apresenta uma superposição de sintomas depressivos e maníacos, com grave irritabilidade, sentimentos de ira e ódio, expressão facial e comportamento incompatíveis com as queixas depressivas (ressonância afetiva), podendo haver demonstrações de violência e auto ou heteroagressividade incontroláveis. Além dos estados mistos, pode acontecer a ciclagem rápida, caracterizada pela mudança abrupta de uma fase para a outra(2).

Enquanto o portador permanece sem os sintomas descritos acima, este se encontra eutímico, ou seja, em remissão. A remissão, porém, não deve ser considerada como sendo apenas a resposta clínica - redução de $50 \%$ dos sintomas observados ou mesmo a ausência de sintomas, mas como a (re)integração funcional do indivíduo em suas atividades de rotina. Nesse sentido, o tratamento do TAB tem por objetivo manter o paciente em eutimia, promovendo a recuperação e evitando a recaída, que é um item de significativa importância, sendo que o risco para tal é maior nos primeiros 4 a 6 meses após a remissão dos sintomas, indicando a não continuidade do tratamento como um dos maiores problemas ${ }^{(1,3)}$.

Estima-se que a bipolaridade pode afetar em torno de $1 \%$ da população geral ${ }^{(1,4-5)}$. Contudo, estudos que consideram o espectro bipolar, indicam uma prevalência entre $5 \%$ e $8 \%$. Em geral, a manifestação dos primeiros sintomas ocorre na adolescência, mais especificamente entre os 18 e 22 anos $^{(1,4)}$. Dessa forma, pode causar uma ruptura do processo de amadurecimento, trazendo prejuízos significativos no âmbito biopsicossocial, dado que nesse período se iniciam vários preparativos para a vida adulta, entre estes, a escolha da profissão, a conquista da autonomia e os relacionamentos amorosos.

Além disso, um estudo realizado sobre as causas de internações psiquiátricas no Município de Ribeirão PretoSP pelo Sistema Único de Saúde constatou que os Transtornos de Humor são a segunda causa de internação hospitalar entre 1998 e $2002^{(6)}$. Também, é considerado pela Organização Mundial da Saúde uma das principais causas de incapacitação no mundo, sendo que indivíduos acometidos têm maiores taxas de desemprego ${ }^{(2,7)}$. Isso fica evidente nos relatos de pacientes, que referem grande sofrimento devido ao prejuízo na capacidade ocupacional e no desempenho social, ao risco de lesão física e suicídio, à perda da credibilidade e confiança, ao medo de recaídas, enfim, às consequências da doença na vida.

causa de internação

O tratamento do TAB consiste em estratégias farmacológicas e não farmacológicas. As primeiras compreendem os medicamentos estabilizadores do humor, tais como o Lítio, Anticonvulsivantes e Antipsicóticos. Estes visam o controle da fase aguda e a prevenção de novos episódios e têm um papel fundamental na reparação da plástica sináptica, compensando uma série de alterações estruturais e funcionais em determinadas regiões do cérebro provocadas pelas recaídas ${ }^{(8)}$.

\section{Estratégias Psicossociais}

Apesar do TAB ser caracterizado por fortes indicadores biológicos e o tratamento farmacológico ser impreterível, é necessário que haja uma atenção psicossocial, considerando que no tratamento estritamente biológico são altos os índices de não aderência e recaídas. Assim, é fundamental associar tratamentos alternativos, que busquem a aplicação clínica do modelo biopsicossocial e a inclusão e valorização da participação dos pacientes e familiares ${ }^{(9)}$.

Inclusive, há evidências crescentes de que o curso do TAB pode ser modificado por meio de abordagens psicoterápicas. 
Estas têm como objetivos o aumento da adesão ao tratamento, a redução dos sintomas residuais, a identificação de pródromos sindrômicos com a consequente prevenção das recaídas, a diminuição das taxas e períodos de hospitalizações e a melhora na qualidade de vida dos pacientes e seus familiares. Além disso, favorecem o aumento do funcionamento social e ocupacional desses pacientes e das capacidades de manejarem situações estressantes em suas vidas ${ }^{(10)}$.

Entre as abordagens não farmacológicas há a Psicoeducação, desenvolvida a partir da década de 1970, como um tratamento adicional ao fármaco, com o intuito de manter o paciente inserido na comunidade ${ }^{(9)}$. Portanto, a psicoeducação caracteriza-se como um dos modelos de reabilitação psicossocial(11), cuja definição compreende o desenvolvimento de um conjunto de programas e serviços que visam facilitar a vida de pessoas com problemas severos e persistentes de Saúde Mental ${ }^{(12)}$.

Assim, percebe-se que o anterior paradigma de assistência psiquiátrica - o asilar - demonstra-se incapaz de atender tais exigências, uma vez que seu cerne é o hospital, a doença mental e o tratamento medicamentoso, desconsiderando o sujeito e seu contexto psicossocial ${ }^{(13)}$.

A abordagem psicoeducativa, portanto, vem ao encontro dos objetivos da Reforma Psiquiátrica, ou seja, a superação gradual da internação nos manicômios através da criação de serviços na comunidade que desenvolvam práticas de prevenção e reabilitação psicossocial ${ }^{(14)}$.

A definição de psicoeducação varia de acordo com os pressupostos e objetivos que os profissionais pretendem alcançar, sendo a abordagem dos aspectos psicológicos o que mais diferencia uma prática clínica de outra. Assim, existem diferentes métodos e técnicas, dependendo do tipo de intervenção e do referencial teórico adotados ${ }^{(9)}$.

De qualquer modo, em geral, a psicoeducação é considerada como uma das principais estratégias para modificar aspectos negativos vivenciados pelos portadores de TAB e envolve fornecer informações aos pacientes e familiares sobre a natureza e o tratamento da doença, provendo ensinamentos teóricos e práticos para que possam compreender e lidar melhor com a mesma. Além disso, tal estratégia tem como objetivo ajudar o portador a melhorar seu insight sobre a doença, lidar com a estigmatização, melhorar a adesão ao tratamento, ensinar os sinais prodrômicos precoces, promover hábitos saudáveis e a regularidade no estilo de vida e evitar o abuso de substâncias ${ }^{(10,15)}$.

A psicoeducação pode ser desenvolvida individualmente ou em grupo, por diferentes profissionais de saúde, desde que capacitados para lidar com as reações associadas ao tema discutido. Isso porque, embora não seja considerada uma modalidade de psicoterapia, aborda aspectos afetivo-emocionais com o intuito de promover mudanças, o que exige certas habilidades do profissional ${ }^{(9)}$.

Certamente, a educação sobre a doença, a identificação e manejo de comorbidades, bem como o estímulo pa- ra mudanças positivas no estilo de vida do paciente e sua família são importantes papéis que devem ser exercidos pela equipe multidisciplinar no tratamento de pacientes com transtornos de humor. Inclusive, deve ser encarada como um dever do profissional frente ao portador, visto que faz parte dos direitos do paciente ser informado sobre sua doença(16-17).

A experiência como membro da coordenação de um grupo de psicoeducação para familiares e portadores confirma os benefícios desse tipo de estratégia. Dessa forma, este trabalho tem por objetivo relatar essa experiência, a fim de contribuir para a prática em saúde mental neste momento de transformações e construção de um novo paradigma - o psicossocial.

\section{MÉTODO}

Este trabalho é parte de um projeto de dissertação de mestrado no Programa de Pós-Graduação da Escola de Enfermagem de Ribeirão Preto/USP (Enfermagem Psiquiátrica), analisado e aprovado pelo Comitê de Ética da Faculdade de Medicina de São José do Rio Preto (Protocolo no 7049/2007). Trata-se de um relato de experiência, com posterior reflexão pautada em um dos componentes do paradigma das práticas em saúde mental ${ }^{(13)}$.

O referido autor denomina as práticas do contexto atual em saúde mental em dois paradigmas básicos: modo asilar e modo psicossocial. Sua análise sobre as contradições existentes entre os dois modelos é feita à luz dos seguintes parâmetros: concepções do objeto e dos meios de trabalho (concepções de saúde-doença-cura e dos meios e instrumentos de seu manuseio); formas da organização do dispositivo institucional (dimensão organogramática); formas do relacionamento com a clientela (diferentes possibilidades de intercâmbio mútuo); formas de seus efeitos típicos em termos terapêuticos e éticos (finalidades político-socioculturais das práticas).

Dentre esses parâmetros, optou-se por considerar as concepções do objeto e dos meios de trabalho, que serão analisadas a partir dos saberes e práticas disseminados em um Grupo de Psicoeducação para familiares e portadores do Transtorno Afetivo Bipolar.

\section{O Grupo}

O Grupo de Psicoeducação para familiares e portadores de Transtorno Bipolar do Humor faz parte do programa de extensão à comunidade oferecido pela Faculdade de Medicina de São José do Rio Preto (FAMERP), que possui os cursos de graduação em Medicina e Enfermagem.

O referido grupo existe desde setembro de 2003 e, inicialmente (até novembro de 2005), era parte do Projeto Prevenção de Recaídas no Transtorno Bipolar do Humor, em parceria com o Grupo de Estudos de Doenças Afetivas (GRUDA) do Instituto de Psiquiatria do Hospital das Clíni-
Grupo de psicoeducação no transtorno afetivo bipolar: reflexão sobre o modo asilar e o modo psicossocial Menezes SL, Mello e Souza MCB 
cas de São Paulo e alguns Serviços de Saúde Mental existentes no município de São José do Rio Preto na época: o Ambulatório Municipal de Saúde Mental; o Hospital Dr. Adolfo Bezerra de Menezes e o Ambulatório de Psiquiatria do Hospital de Base.

O grupo surgiu como uma estratégia para diminuir as frequentes internações de indivíduos portadores de Transtorno Afetivo Bipolar (TAB), bem como para suprir a falta de suporte oferecido aos mesmos e aos seus familiares. Em geral, esses tinham pouco ou nenhum conhecimento sobre a doença, seus tratamentos, bem como sobre como prevenir as recaídas e, consequentemente, as reinternações.

Mesmo após o término do projeto citado esse grupo teve continuidade na FAMERP devido ao interesse dos profissionais envolvidos, bem como a pedido dos próprios familiares e portadores de TAB. Nessa época, eu já não atuava mais como enfermeira no Hospital Psiquiátrico do município, mas como uma das docentes da Disciplina de Enfermagem Psiquiátrica e Saúde Mental do Curso de Graduação em Enfermagem da FAMERP.

\section{Funcionamento do Grupo}

Trata-se de um grupo aberto, com encontros mensais, participando, em média, trinta pessoas (entre familiares e portadores), que são convidadas por meio de anúncios de jornais, cartazes distribuídos nos Serviços de Saúde Mental do município, bem como pessoalmente pelos profissionais que participam do grupo ou que são nossos colaboradores.

É programada para cada encontro uma palestra proferida por algum profissional, de acordo com o tema agendado e sua área de conhecimento (enfermeiro, médico psiquiatra, psicólogo, nutricionista, educador físico, advogado e outros).

Quanto aos temas abordados nas palestras, esses são sugeridos pelos próprios participantes, ao responder um questionário de avaliação dos encontros, que é aplicado anualmente. Em geral, os temas incluem a caracterização da doença em suas diferentes fases; fatores causais e desencadeantes; tratamentos farmacológicos e não farmacológicos; gravidez; detecção precoce dos episódios maníacos e depressivos; álcool e outras drogas; prejuízos psicológicos, sociais e econômicos; técnicas de gerenciamento do estresse; como lidar com um familiar que é portador; estratégias para conviver com as mudanças no estilo de vida no dia-a-dia e melhorar a qualidade de vida (alimentação e atividade física); direitos do portador e familiar; entre outros.

Após a palestra, há um depoimento voluntário de um familiar ou portador, cujo objetivo é a troca de experiências e o intercâmbio de encorajamento. Além do depoimento, é marcante a participação dos presentes em expressar seus pensamentos e/ou dúvidas acerca do tema e estas últimas são esclarecidas pelos profissionais pre- sentes. Os encontros são finalizados com um lanche, momento esse que favorece a aproximação entre familiares/ portadores e os profissionais, contribuindo, assim, para o fortalecimento do vínculo entre os mesmos.

Atualmente, a coordenação do grupo é composta por três enfermeiras, sendo que eu e mais uma delas atuamos como docentes da FAMERP e a outra é enfermeira num Centro de Atenção Psicossocial para dependentes de álcool e outras drogas.

A coordenação do grupo envolve as seguintes ações: proferir palestras; mediar as discussões; esclarecer dúvidas; criar estratégias que favoreçam o aprendizado, bem como as trocas entre os próprios participantes e destes com os profissionais presentes; montar o cronograma, estabelecendo o local, o horário e a frequência dos encontros, bem como os temas das palestras, de acordo com as necessidades e sugestões dos integrantes e escolhendo os profissionais palestrantes, conforme os objetivos de cada encontro; verificar se os objetivos do grupo estão sendo alcançados e o nível de aproveitamento/satisfação dos participantes, a fim de fazer as adaptações necessárias.

Como facilitadoras nesse processo de psicoeducação, buscamos desenvolver uma capacidade interacional que propicie criar vínculos entre nós e os integrantes e entre $\mathrm{si}$, a fim de que haja trocas construtivas.

\section{Análise das Concepções do Objeto e dos Meios de Tra- balho de acordo com o paradigma das práticas em saú- de mental(13)}

Entende-se por concepções do objeto e dos meios de trabalho as concepções de saúde-doença-cura e os meios e instrumentos de seu manuseio. Nesse sentido, no modo asilar privilegia-se o tratamento medicamentoso, uma vez que a ênfase está nas determinações orgânicas dos transtornos psiquiátricos, em detrimento da subjetividade do sujeito. Isso fica evidente na desconsideração do indivíduo como participante do tratamento, visto que nessa concepção o que importa mesmo é a ação do remédio. Dessa forma, o meio familiar e social não são levados em conta, excluindo a família das práticas de tratamento ou considerando-a apenas como um coadjuvante.

O trabalho no modo asilar, mesmo que seja multiprofissional, é fragmentado, similar ao modelo da divisão do trabalho no capitalismo. No hospital psiquiátrico - instituição típica desse modelo - o paciente é atendido pelo psiquiatra, que define o problema (doença - diagnóstico), estabelece o tratamento (medicamentoso) e determina se haverá necessidade de ocorrer ações de outros especialistas. Assim, não há um trabalho em equipe, muito menos consideração do paciente como participante desse processo. Portanto, no modo asilar privilegia-se o hospital como local de tratamento e o trabalho é centrado no saber médico, considerando os demais profissionais como secundários - paramédicos, não-médicos. 
Já no modo psicossocial, o foco das intervenções é o sujeito como um ser político e biopsicosociocultural, levando em consideração, portanto, a pertinência do indivíduo a um grupo familiar e social, o que determina que se deva trabalhá-los como agentes das mudanças buscadas.

Além disso, os meios de tratamento visam a autonomia do sujeito, que ele não apenas sofra os efeitos dos seus conflitos, mas que se reconheça como um dos agentes envolvidos nesse sofrimento e, a partir disso, como um disparador de mudanças.

Analisando o grupo de psicoeducação sob esse prisma (objeto e meios de trabalho), percebo que o mesmo aproxima-se do modo psicossocial, uma vez que seu foco é o indivíduo, considerando os aspectos políticos e biopsicossociais. Tanto que o grupo de psicoeducação não se restringe ao portador, mas, também, ao seu grupo familiar, tornando os familiares não meros expectadores, mas integrantes fundamentais dessa abordagem.

A todo momento, buscamos a participação ativa dos sujeitos, conscientizando-os do papel que têm em relação a sua existência-sofrimento, como protagonistas das mudanças no estilo de vida, prevenção de recaídas e reinserção social. São nesses encontros que eles discutem com seus pares (portadores e familiares) novas possibilidades, como a participação no Conselho Municipal de Saúde, a criação e/ou participação de associações de usuários de saúde mental, entre outras.

Isso porque o processo educativo deve ser de natureza dialógica, baseado no contexto de vida das pessoas, dos seus cotidianos, das suas experiências, visando a libertação do indivíduo para que possa ser um sujeito social capaz de intervir em sua vida e no ambiente, conquistando, assim, sua autonomia ${ }^{(18)}$.

Por meio dos depoimentos dos participantes, percebemos o quanto o conhecimento sobre o TAB pode favorecer o comprometimento com o tratamento e, assim, o quanto a psicoeducação exerce um papel importante para isso. Ao compreenderem a dinâmica da doença, sua natureza cíclica e crônica, bem como os prejuízos advindos das constantes recaídas, passam a se conscientizar de que são portadores de um transtorno mental e que, necessariamente, precisam realizar o tratamento da forma adequada.

Ao mesmo tempo, notamos que eles se dão conta de que podem ser participantes ativos nesse processo e que essa adesão ao tratamento Ihes propiciará uma meIhor qualidade de vida. Nesse sentido, mencionam como benefício dessa conscientização da doença e adesão ao tratamento a possibilidade de terem uma vida normal, apesar do transtorno, gerenciando suas vidas ao invés de viverem à mercê dos altos e baixos da doença. No que diz respeito a isso, retomaram suas atividades cotidianas, tais como o trabalho secular e as responsabilidades domésti- cas, atividades estas que estavam intensamente comprometidas na vida de alguns deles. Mencionam, também, a possibilidade de necessitarem de menos internações psiquiátricas, uma vez que aprendem a lidar melhor com a doença e suas consequências. Além disso, citam que a participação no grupo favoreceu o aumento na auto-estima, contribuiu para se sentirem mais felizes e os ajudou a conviver da melhor maneira possível com sua nova situação de vida.

Quanto aos meios de trabalho, buscamos o trabalho em equipe, havendo a participação de enfermeiros, psiquiatras, psicólogos, nutricionistas, professores de educação física, advogados, entre outros. $\mathrm{E}$, diferente do modo asilar, não há um ator principal (psiquiatra), nem temos como instituição típica o hospital. Isso porque atuamos fora do hospital, na comunidade, onde, por meio da psicoeducação, buscamos não somente a supressão dos sintomas mas a prevenção das recaídas com vistas à diminuição das internações e subsequente reinserção social.

Assim sendo, procuramos ir além da consideração sobre a doença (Transtorno Afetivo Bipolar), ao passo que buscamos a subjetividade singularizada, que a cada encontro é expressa por meio das falas, questionamentos e depoimentos dos participantes. Nesses depoimentos, não se focalizam apenas os sintomas e recaídas, mas o contexto de vida desses sujeitos, seus sofrimentos, frustrações, objetivos alcançados, perdas, sonhos, desejos e planos de vida.

No paradigma asilar, os sujeitos assumem uma postura passiva, à mercê das intervenções dos profissionais, ou seja, meros depositários daqueles tidos como sãos e detentores do conhecimento. Já no modo psicossocial, espera-se que os sujeitos se administrem e é isso, também, que buscamos no grupo de psicoeducação para familiares e portadores de TAB.

\section{CONCLUSÃO}

Embora o TAB tenha fortes características biológicas e, portanto, o tratamento farmacológico seja fundamental, este por si só demonstra-se insuficiente, dado a complexidade do sujeito portador e seu contexto biológico, psicológico, social, político e cultural. Nesse sentido, uma abordagem que contemple tais dimensões, certamente, terá mais possibilidade de eficácia.

Partindo desse pressuposto, a reflexão sobre o modo asilar e o modo psicossocial no que tange às concepções do objeto e do modo de trabalho possibilitou situar o nosso grupo de psicoeducação para familiares e portadores de TAB como uma estratégia do modo psicossocial. Isso porque o conhecimento e a vivência experimentados nesses encontros favorecem a autonomia dos sujeitos, que têm maiores chances de se posicionarem frente às dificuldades que lhes sobrevém na sua existência-sofrimento. 


\section{REFERÊNCIAS}

1. Souza FGM. Tratamento do transtorno bipolar: eutimia. Rev Psiquiatr Clin. 2005;32(1):63-70.

2. Moreno DH, Almeida KM. Quadro clínico dos subtipos do espectro bipolar. In: Moreno R, Moreno DH. Da psicose maníaco-depressiva ao espectro bipolar. São Paulo: Segmento Farma; 2005. p. 141-69.

3. Stuart GW. Respostas emocionais e transtornos de humor. In: Stuart GW, Laraia MT. Enfermagem psiquiátrica: princípios e prática. 6a ed. Porto Alegre: Artmed; 2001. p. 380-437.

4. Kessler RC, Rubinuw DR, Holmes C, Abelson JM, Zhao S. The epidemiology of DSM-III-R bipolar I disorder in a general population survey. Psychol Med. 1997;27(5):1079-89.

5. Angst J, Gama A, Benazzi F, Aidac V, Eich D, Rossler W. Toward a redefinition of subthereshold bipolarity epidemiology and proposed criteria for bipolar I, minor bipolar disorder and hypomania. J Affect Disord. 2003;73(1-2):133-46.

6. Silva EC. O Sistema de Informações Hospitalares do Sistema Único de Saúde: subsídios para o uso de dados secundários em psiquiatria e saúde mental [dissertação]. Ribeirão Preto: Escola de Enfermagem de Ribeirão Preto, Universidade de São Paulo; 2004.

7. Lima MS, Tassi J, Novo IP, Mari JJ. Epidemiologia do transtorno bipolar. Rev Psiquiatr Clin. 2005;32 Supl 1:15-20.

8. Frey BN, Fonseca MMR, Machado-Vieira R, Soares JC, Kapczinski F. Anormalidades neuropatológicas e neuroquímicas no transtorno afetivo bipolar. Rev Bras Psiquiatr. 2004; 26(3):180-8.

9. Andrade ACF. A abordagem psicoeducacional no tratamento do transtorno afetivo bipolar. Rev Psiquiatr Clin. 1999;26(6):1-8.
10. Knapp P, Isolan L. Abordagens psicoterápicas no transtorno bipolar. Rev Psiquiatr Clin. 2005;32(1):98-104.

11. Guerra AMC. Reabilitação psicossocial no campo da reforma psiquiátrica: uma reflexão sobre o controverso conceito e seus possíveis paradigmas. Rev Latinoam Psicopat Fund. 2004;7(2):83-96.

12. Pitta AMF. O que é reabilitação psicossocial no Brasil, hoje? São Paulo: Hucitec; 1996. Reabilitação psicossocial no Brasil; p. 19-26.

13. Costa-Rosa A. O modo psicossocial: um paradigma das práticas substitutivas ao modo asilar. In: Amarante $\mathrm{P}$, organizador. Ensaios: subjetividade, saúde mental, sociedade. Rio de Janeiro: FIOCRUZ; 2000. p.141-68.

14. Rotelli F, Leonardis O, Mauri D. Desinstitucionalização. São Paulo: Hucitec; 1990. p. 17-21.

15. Miasso Al. "Entre a cruz e a espada": o significado da terapêutica medicamentosa para a pessoa com transtorno afetivo bipolar, em sua perspectiva e na de seu familiar [tese doutorado]. Ribeirão Preto: Escola de Enfermagem de Ribeirão Preto, Universidade de São Paulo; 2006.

16. Machado-Vieira R, Santin A, Soares JC. O papel da equipe multidisciplinar no manejo do paciente bipolar. Rev Bras Psiquiatr. 2004;26 Supl 3:51-3.

17. Colom F, Vieta E. Melhorando o desfecho do transtorno bipolar usando estratégias não farmacológicas: o papel da psicoeducação. Rev Psiquiatr Clin. 2004;26(1):47-50.

18. Ruiz VR, Lima AR, Machado AL. Educação em saúde para portadores de doença mental: relato de experiência. Rev Esc Enferm USP. 2004;38(2):190-6. 